\title{
COMMUNICATION OF TWO CONTROLLER AREA NETWORK SEGMENTS THROUGH WIRELESS SENSOR NETWORK
}

\author{
Shanthi Makka ${ }^{*}$ and B.B.Sagar ${ }^{2}$ \\ ${ }^{1}$ Computer Science and Engineering, BIT, Mesra (Ranchi), India \\ ${ }^{2}$ Computer Science and Engineering, BIT, Mesra (Ranchi), India \\ ${ }^{1}$ shanthi.makka@gmail.com, ${ }^{2}$ drbbsagar@gmail.com
}

\begin{abstract}
In the current scenario, the wireless sensor networks are nothing but communication, computation, and sensing can be combined into a small-scale device. These devices provide connectivity to reach out the cyberspace to the real world or physical world with the help of mesh networking protocols. The connectivity in the mesh network consistently finds or searches for the feasible communication path by moving data from one to another while searching for its target. When any single device shows minimum capability, then the configuration of many devices shows rigorous prospects in new technology. The main functionality of Wireless Sensor Networks is always relies on the formation of small nodes, which can acquire and built by themselves. The Implementation scheme for such mechanism scales from real-time tracking to supervision of substantial states to pervasive enumerates environments, to the observation of the soundness of structures or equipment. The Controller Area Network (CAN) is precisely implanted with many distributed realtime controlled applications and automation applications in industries and robotics. But still, there is a problem with the CAN based distributed control system as the size of the dispersed area is small and also devoir to communicate with other wireless networks and with distant $\mathrm{CAN}$ piece. In this paper, we have recommended a new system, which makes use of inter-working components with wireless support to connect CAN portions by using Wireless Sensor network with particular protocols.
\end{abstract}

Keywords- Controller Area Network (CAN), Wireless Sensor Network, Interfacing Bridge-Device, Mobile CAN, NS-2

\section{INTRODUCTION}

Currently, primary Communication network can be classified into four groups [12]:

- Wireless Local Area Network (WLAN)

- 3G or 4G Cellular Network

- IP Core Network/Internet

- Ad-hoc Personal Area Network

The general utilization of specified networks is to transmit and receive data content either in the form of text, audio, and video. However, the future of these networks is much bright in industrial automation as monitoring and controlling various industrial plants.

Received: September 15, 2019

Reviewed: November 18, 2019

Accepted: December 3, 2019 
In addition to the above, there is a different type of network is Controller Area Network (CAN), its primary focus as a communication network between the control units in vehicles. As we all know the vehicles or Robots or Aerospace machines etc. are exploring CAN protocol as much as possible because it is simple and real-time but distributed controlled behavior. But one machine, if it is mobile, cannot communicate with another machine by using only CAN. It requires at least one wireless media to perform its connection and session maintenance during mobility. So different types of such wireless technologies are utilized as [3]:

- Wireless Personal Area Network

- Bluetooth

- Infrared

- Wireless Local Area Network

Network devices are developed to perform their successful implementation. As per observation based on some academic surveys, it is proved that Wireless Sensor Network is better in many aspects in terms of performances as compared to other networks. So accordingly it should have the highest priority among all other technologies that never use dedicated wires and cables [11]. In this paper we have explained Wireless Sensor Network and CAN briefly in section 3. It is also mentioned in section 2 that how interconnection between two CAN base network portions with the help of Wireless Sensor Network is possible.

\section{MOTIVATION}

Due to advanced technologies available in the industries, the erection of low-cost sensors and small sensors is accessible very easily in both technically and economically. In the implementation of intelligent transportation systems, with the advancement of mobile networks, the wireless sensor networks, Internet of Things (loT), and vehicular networks have progressively became as one of the most adequate access. The sensing electronics [21] amplification influences the background enmeshed to the environment encompassing the sensor and alters them into an electric signal. Processing such a signal reveals some features about objects posted and/or events proceeding in the proximity of the sensor. A huge quantity of these insubstantial sensors can be structured in a mass number of operations that depend upon ignored applications. A Wireless Sensor Network abides of thousands of sensor nodes and these nodes have dexterity to broadcast to either among each other or precisely to an extraneous base-station.

A huge estimate of sensors permit the sensing concluded huge topographical segments of preeminent veracity. Essentially, each sensor node encompasses sensing, processing, transmission, mobilizer, location discovery system, and power units and mobilizer is an optional component. The sensor nodes are constantly distributed in sensor farmland that an area where the sensor nodes are extended. Sensor nodes integrate among themselves to contribute the finest report about the physical aura. Each sensor node supports its accord on its objective, the blockhead forthwith has, and its knowledge of its cipher, connection, and energy resources. Each of these distributed sensor nodes has the competence to gather and direct data either to other sensors or revert to an external base station or stations. The controller area network minimizes electrification since it is a shared control and this guarantees enhancement of the system achievement, its support multiple masters and multicast lineaments. The CAN bus has a peak length of 40 meters. The CAN accommodates the capability to work in various electrical prospects. The enlarged CAN improve the galvanized to establish impend to enact elicitation between CAN and WSN. 


\section{INTEGRATING CAN PIECES OVER WIRELESS SENSOR NETWORK}

\subsection{CONTROLLER AREA NETWORK (CAN)}

The Controller Area Network Bus is a bus protocol in which the nodes communicate through messages and it is frequently used in automobiles. The CAN [20] bus protocol is drained out to empower coordination between discrete Electronic Control Units (ECUs), such as Powertrain Control Module (PCM), Airbags, Cruise control, an Engine Control Module (ECM), Antilock Brakes, Electric Power Steering, Audio Systems, Windows, Doors, Adjustment or Mirror, Battery Recharging Systems for Hybrid and Electric cars, and various number of features in automated vehicles.

Controller Area Network has essentially used the serial bus system in Distributed Control Systems. In the late 1980s, Robert Bosch GmbH from Germany has developed to employ only motor vehicles with CAN license. The International Standardization Organization (ISO) and the Society of Automotive Engineers (SAE) standardize controller Area Network and this protocol uses Physical Layer and Data Link Layer of ISO-OSI model $[1,2,4,8]$. A CAN is a vigorously used in vehicle bus standard, dedicated to permitting microcontrollers and peripherals to transmit data from one to another without any host computer and just by using a common cable. There are many protocols available for CAN and it is also most widely used in motor vehicles, utility vehicles, health care equipment, automation of houses and household appliances, automation of offices and industries. The availability of low-cost protocol devices got increased because of a large volume of production in both automotive and industrial markets.

The important features of CAN are: minimal cost physical medium, serial interface with very high speed, minimum data length, quick response time, multi-master peer-peer communication, efficient way for error detection and correction, and the nodes attached to bus uses CSMA/CD+CR as the mediation approach to capacitate its passage to the bus. The twisted-pair cable to communicate in CAN uses the maximum speed of data transfers 1 MBPS. The data transfer rate is 1 Mbps up to 30 meters and it is reduced to $100 \mathrm{Kbps}$ up to 100 meters. Due to its real timeliness [1,3,4], CAN shows $100 \%$ bandwidth utilization. CAN (version 2.0) have two distinct standards; CAN 2.0A and CAN 2.0B is also called as extended CAN. CAN 2.0A standard use 11 bits as identity of a node, and the CAN 2.0B or extended CAN uses 29 bits as node identity. A CAN message comprises $0-8$ bytes of data and 44 bits of information regarding controlling of vehicles for protocol and these 44 bits consist of identifier, acknowledge CRC, and synchronization and so on.

\subsection{WIRELESS SENSOR NETWORK}

The Wireless Sensor Networks (WSNs) are essentially used in information technologies [18] of advanced networking and computing stages. Today's network computing functions are confronted with a steep appeal of persuasive network services. Functional network influence is fundamental to the satisfaction of the customer such as in mobile-networks and cloud computing ambiances. Thousands of applications come into mind once people can understand the capabilities of a wireless sensor network. It sounds agnate a candid brew of modern technology. Nonetheless, connecting sensors, radios, and CPUs into an ample wireless sensor network anticipate an exhaustive amiable of both performance and restraint of each of the prerequisite hardware factors, as well as an embellished understanding of modern networking technologies and distributed systems theory. Each definite node must be invented to furnish the position of savages crucial to absorb the pertain web that will emerge as they are not substantial, while battle firm necessary of size, cost, and power application. 
A substantial challenge is to outline the entire system applications down to particular device potential, essential and actions. To make the wireless sensor network perception more accuracy, construction must be arranged that [19] consolidate the prevision functions out of the masked hardware effectiveness. In specific, a Wireless Sensor Network (WSN) will be used to assemble real-time traffic and concrete data to construct vivid and presaging models to design the finest routes to decrease road surfeit and thereupon-metropolitan pollution.

\subsection{SENSOR NETWORK APPLICATION CLASSES}

In this section, we discussed three application classes: the assemblage of environmental data, supervising of security, and capturing of the sensor node. We can categorize the wireless sensor network into one of these classes.

\subsubsection{ASSEMBLAGE OF ENVIRONMENTAL DATA}

To determine direction and credence in an environmental data assemblage, researchers and scientists compile data i.e., distinct sensor examinations from a group of points in an environment for a specific era of time. Broadly these scientists assemble data from hundreds of point's acceleration over the area and then investigate this data offline [8,9]. The scientist would adopt to cluster data over many months or years in an establishment to find data for long-term and periotic direction. To preserve the performance of data it must be possessed at typical intervals and nodes must be at their positions. At the network level, anticipating the huge number of nodes are repeatedly disseminate data to boost to an establishment of base stations, which accumulates the data applying typical accession allocate the environmental data accumulation, and these networks requires low data rates and fiercely high lifespans.

\subsubsection{SUPERVISING OF SECURITY}

It is the second category of a sensor network application that consumption of nodes that are posted at a consistent area encompassing a situation that continuously monitored one or more sensors to pinpoint an aberration. Custody supervising bound a primary diversity and environmental observes that a security network never collects any data. This has a consequential draft on the construction of an optimal network. In continuous intervals, every node must observe the condition of its sensors and the communication of the data has to be done at least once there is a security contravention.

The essential appeal of the system [17] is censorious and conclusive enunciation of alarm messages and "report by exception" networks do allusion and in inclusion, to that, it is destitute to be addicted about current and working of each node in the network. If any node is stalled or declined, it would invent a violation of security that should be described. In security tracking applications, the nodes are accurate for conclusive the condition of each other; subsequently, the composition of the network has to be done. The highest topology of a security-tracking network will arrive particularly from that of the datagathering network. One access is to have each node be allotted to a neighbor node or companion that takes the burden to an article if a node is not activity.

\subsubsection{CAPTURING OF SENSOR NODE}

A third module draft intermittently contemplates for a sensor network is the unmasking of consecutive objects over a part of space scrutinize by a sensor network. There are many viewpoints where one could like to discover the commendable assets or personnel. Current backlog control systems analysis to monitor objects from the checkpoint that objects lastly driven through and also document their location. However, these systems cannot be passed down to get the present position of an object and in wireless sensor 
networks, a tiny sensor node can be targeted to monitor objects by simply emblem. The sensor node will be expelled as it handles through a chunk of sensor nodes that are developed in the resources at accepted positions. Comparatively, then sensing environmental data, these nodes will be biased to sense the RF messages of the nodes fasten to definite objects; supplementary these nodes can be employed as effective tags that distributed the habitation of a mechanism.

The location of the chased objects can be registered in the database in coordinate with the set of nodes at already known positions that can give us the information where an object is presently commenced, which is not easy where it was antecedently scanned [13].

\subsection{SYSTEM EVALUATION METRICS}

In this section, we are going to inspect how to evaluate Wireless Sensor Networks through evaluation metrics after the establishment of the set of application scenarios that we addressed in previous sections. To proceed further in this aspect, we need to keep in mind that the high-level objectives of the network deployment, the proposed utilization of the network, and the key dominances of Wireless Sensor Networks bygone current technologies.

\subsubsection{LIFETIME}

Condemnatory to any Wireless Sensor Network implementation is a conventional lifetime. The primary focus of both the Assemblage of Environmental Data and Supervising of Security scenarios is to have nodes implanted in the territory, let unattended for months or years. In Sensor Network, the main prescribed component for the lifetime is Energy supply. To increase the efficiency of the total time of a lifespan of a network, every node must be adequate to take care of its local supply. In the decision technique of the lifetime of given energy supply, radio power consumption shows a important act. In a Wireless Sensor Node, the radio consumes the constant preponderance of the system energy. This power decay can be shrink over recede the transference yield power or by minimizing the radio duty cycle. Both ways are accomplice deny with other system metrics.

\subsubsection{COVERAGE}

In a wireless network, coverage is the primary evaluation cadent. It is consistently fortunate to have the expertise to expend a network over a huge physical area. This can automatically breakthrough a value of a system to the end-user. It is pre-requisite to keep in mind that the ambit of the network is not equal to the bounds of the wireless transmission links being used. Multi-hop transmission approaches can increase the scope of the network well further the scope of the radio technology discarded. In theory, they can expedite the network scope forever. A user must be assured that the network technology being used is enough and has intensified to shelter his probable stipulation. Strengthening the various nodes in the system will force one of the period or tolerable sample rate. More sensing points will signifies more data to be transmitted, which will boost the power deficiency of the network.

\subsubsection{COST AND EASE OF DEPLOYMENT}

A main influence of Wireless Sensor Networks is depending upon on the serenity of formation. Conservationist and development peasants furnish networks cannot be recognizable to distinguish the guarded networking and transmission system at work inside the Wireless Network. For system categorization to be strong, the Wireless Sensor Network must implement itself. It must be feasible for nodes to be posted all over the 
background by a new person and have the system directly work. Correctly, the system would commonly compose itself for any vulnerable concrete node induction.

\subsubsection{RESPONSE TIME}

Exclusively in our apprehension application outline, analytical enforcement metric is time in the system. Dismay must be gesticulating expeditiously when an illusion is dignified. In disregard of low power operation, nodes must be capable in having demanding, high-priority messages published beyond the network as soon as possible. While these events will be random, they may terminate at any time without any prior notice. Response time is also ambitious when concrete control is developed down to curb factory machines and devices. The construction of these systems is feasible if the response time of systems guarantees the approachability. The effectiveness to have low response time contend with many of the techniques passed down to rise the lifetime of the network.

To accelerate the lifespan of a Computer Network, we can make nodes to work in their radios for assertive periods of time. If a node merely moves on its radio once per minute to communicate and acquire the data, it would be arduous to rectify the application appeal for a response time of a security system. Estimation of nodes that are animate all the time can increase response time. These nodes can acquire for the alarm messages and progress them sagged a routing fortitude when necessary. This, however, decline the relaxation of distribution for the system.

\subsection{INTERCONNECTING MODULE (CAN AND WIRELESS SENSOR NETWORK)}

The interconnecting module here is devised as an internetworking unit that is a niceperformance device applied for maintaining the connection between different or similar types of Networks. If both interconnected Networks are of similar types then a passthrough forwarding process is adequate while we have to formulate some translation procedures also if Networks are having dissimilar characteristics. So here the proposed interfacing device must perform learning, translating, storing and forwarding activities to establish connected communication between two CAN 2.0 based network pieces via Wireless Sensor Networks. Encapsulation is the technique applied here for sending a CAN message in Wireless Media. It is fortunate that the CAN message of 108 bits can easily expedient into a Wireless Sensor Network, which has the frame size of 448 bits. Now, this packet can traverse on wireless media while carrying CAN message along with it. On another end, again De-encapsulation procedure will extract the CAN message for further use.

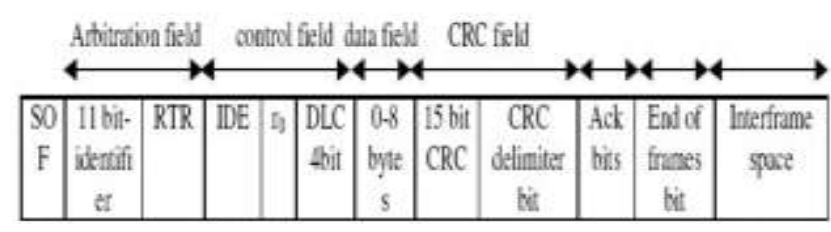

Fig. 1 CAN Standard Frame format [3]

Now to bridge the gap between two CAN nodes based network pieces an inter-working bridge type device is proposed. Its Network Model is depicted in below Figure. 


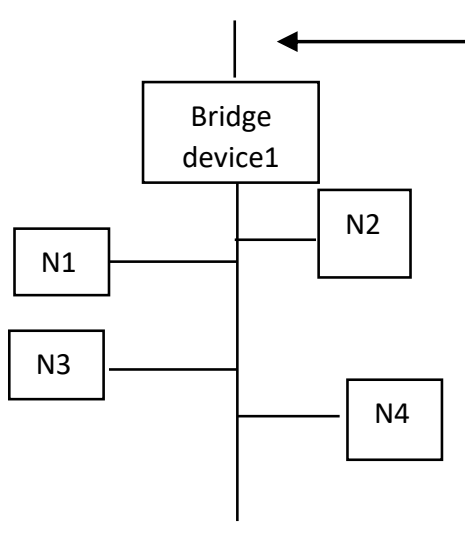

CAN Piece \#1

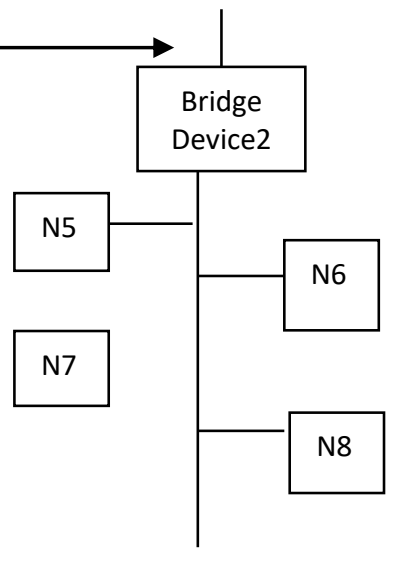

CAN Piece \#2

Fig. 2 Network Model [22]

As per Fig. 2 each CAN piece has four normal nodes and one wireless-based bridge device as a fifth node in corresponding CAN bus is attached. Each normal node in CAN has its priority equal to its node number only as: Node 1 (N1) has priority as P1 and so on. The functional Block diagram of the Bridging Module is defined in Fig. 3. It can be described as CAN and Wireless Sensor Networks data Interfaces have transmitters as well as receivers to get and send data to/from CAN or Wireless Network media buses CAN and Wireless Sensor Network receiver storage blocks are FIFO buffers to store corresponding interface's messages. A matching Table [14-16] is generated after applying the flooding mechanism when it was empty. This approach is useful to discriminate the type of destinations required for a particular message accordingly we can distribute messages in such a way that the local messages can go to one CAN and remote messages to another wirelessly connected CAN.

To develop a wireless interface unit or device it is very necessary to take benefits of both real-time capabilities of CAN and mobile controlling power of Wireless Local area Network. If we remove CAN and use WLAN in place of that then Noise and other environmental obstructions will affect the performance badly. Similarly, if we remove WLAN and use CAN only then there is no possibility to achieve mobility during communication-related functions. So it is urgent to create a combination of both and it should be always within the acceptable limits of any performance standards established by ISO/CMM/IEEE type of organization.

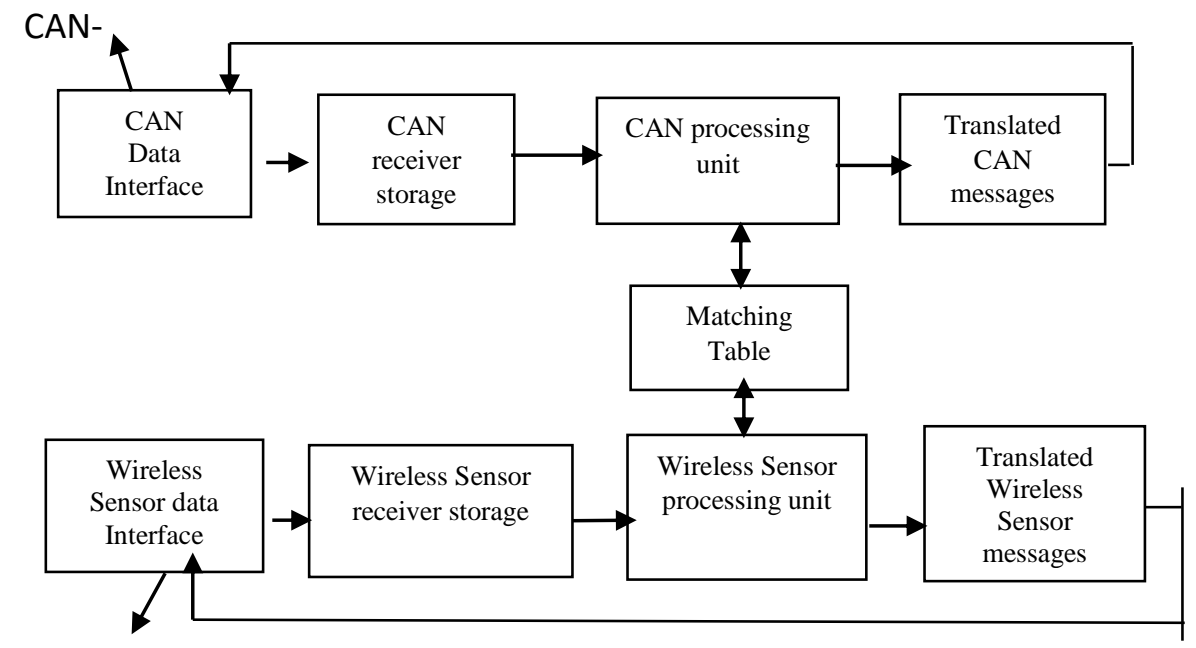

Wireless Sensor Network

Fig. 3 Bridging Module [17] 
The identifiers in CAN messages are also correlated by CAN processing unit by using this Table. So this table is significantly controlling the processing. The CAN processing unit performs encapsulation-based translation of remote destined messages then stores them in FIFO2 from where messages are sent to Wireless Sensor Network data interface while filters local messages. Wireless Sensor Network processing unit performs [17] Deencapsulation-based translation to extract the CAN message then it also compares the identifier of a message with the help of Table to confirm its destination. FIFO3 buffer contains all the messages received by Wireless Sensor Network matched with the table, now these can be further transferred to the corresponding CAN piece. So we have to transfer data in two directions: CAN to Wireless Sensor Network and Wireless Sensor Network to CAN. While doing so we have to follow the above functional block diagram of an interfacing bridge module and We have to verify continuously whether our buffers are already having someplace to keep the next message data or they are full. If they are full we have to confirm the corresponding data-accepting block is functioning properly or not.

\section{NS2: OVERVIEW}

We have implemented or simulated our concept using Network Simulator-2 (NS-2). It is a packet-level network simulator and also follow event-driven methodology refined as part of the Virtual Internet Test (VNIT) project. This was teamwork of a mass number of organizations that are UC Berkeley, and AT\&T Laboratories. The Network Simulator-1 was originated in the year 1995 and version 2 published in the year 1996. Especially, the Version 2 deals with a scripting language, Object-oriented Tcl (OTcl). It is an opensource software package accessible for Windows 32 and Linux Operating Systems.

The features extendable in NS-2:

- It can figure out Pursuance of current network protocols.

- It can also Find the efficiency of new network protocols before use.

- It can Amble large-scale experiments.

- It can Replicate a variety of IP networks.

\section{RESULTS}

The performance of the Network is intensely compassionate to the Data transmission rates of both CAN and WLAN. The transmission rates are 1MBPS and 11MBPS of CAN and WLAN respectively and also depend upon the priority-based message distribution that is promoted by CAN. The total number of maximum possible messages is distributed as follows in Fig. 4:

1- Local messages in CAN 1

2- Remote messages crossing Bridge

3- Local messages in CAN 2

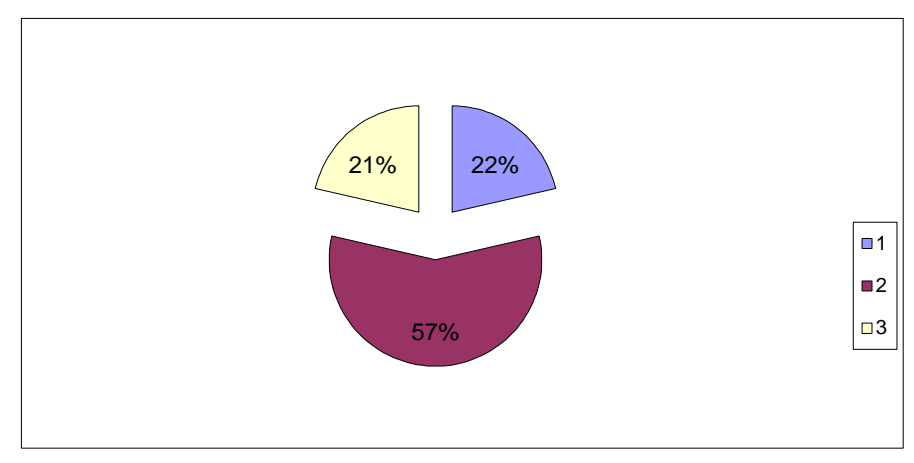

Fig. 4 Possible message distribution 
Now following two figures show graphical representations of CAN to WLAN and WLAN to CAN message passing by using one procedure implemented in $\mathrm{C}++$ and called in NS2 code when the ratio of messages is as previously mentioned verses average execution times:

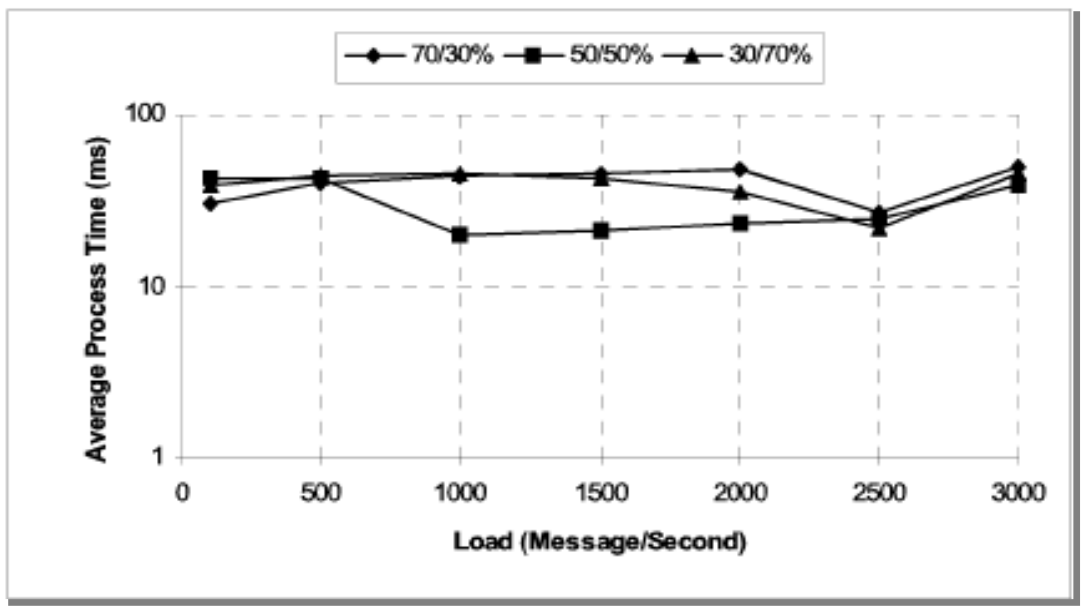

Fig. 5 The CAN and $802.11 \mathrm{~b}$ WLAN bridge model

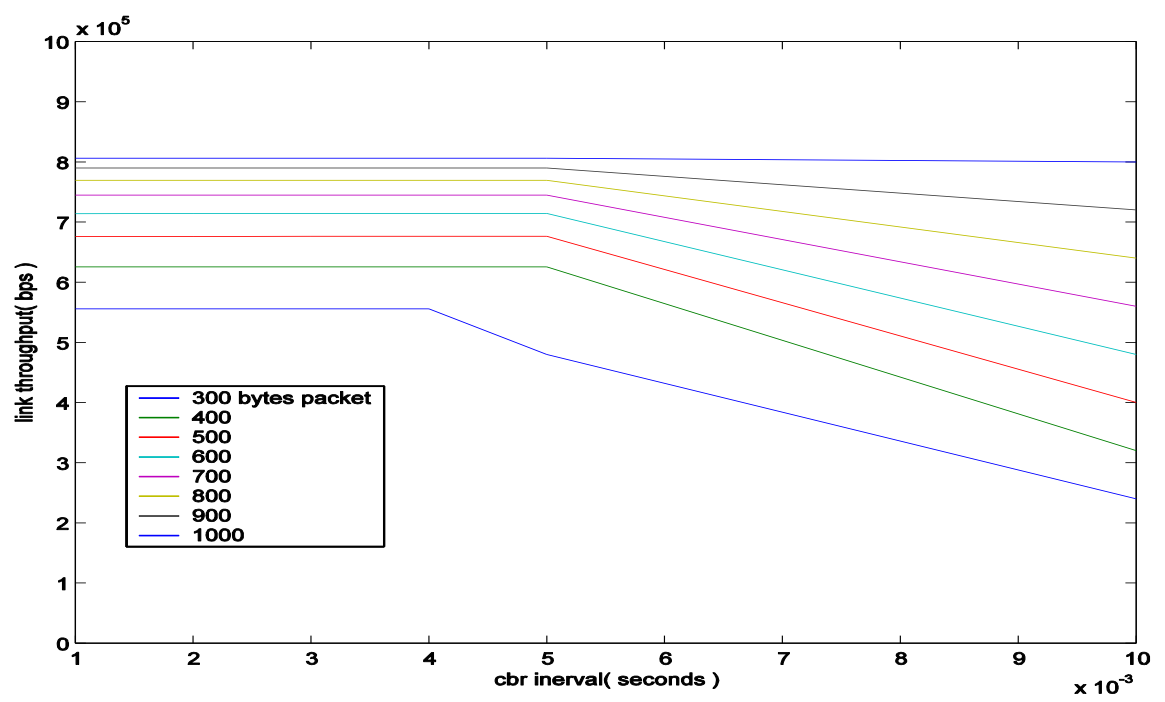

Fig. 6 Throughput between 2-nodes in $802.11 \mathrm{b6}$. FUTURE DIRECTION

An IoT system can be replaced by the WSN because, In an IoT system; the information sent by the sensors will directly to the Internet. For example, if we want to monitor the temperature of the body of water, we can directly use a sensor, and in this situation, the data is immediately broadcast to the Internet, then the server process the data sent and it can be further communicated to a front-end interface. Contrarily, in a WSN, there is no direct communication with the Internet. Alternatively, the different sensors linked to some sort of router or central node, then the person may send the data from the router or central node as they see fit. That means an IoT system can employ a wireless sensor network by connecting with its router to collect data. So we can think of a wireless sensor network as more of an accumulation of sensors or "a big sensor", unlike IoT.

\section{SUMMARY AND CONCLUSION}


To summarize this research work, the functional model of the designed CAN-WLAN interconnecting bridge type device has four major phases of execution. Initially, CAN messages will flow from CAN to WLAN then encapsulated message will flow in wireless media in the second phase than in the third phase after filtering decision CAN message will be extracted from WLAN packet and in last CAN message will flow towards its destined CAN node.

The results: as processing time as well as end-to-end message-delay appeared are also under the limitations of standards defined by the Society of Automotive Engineers Benchmark (SAE) for a CAN. As at last as a concluding remark, I can say that I have completed a very important portion of my project by realizing partial Controller Area Network, full Wireless Area Network and one combination of wired-cum-wireless network with the help of very optimistic and progressive Simulation Environment provided by NS2 software on windows with CYGWIN. As per further progress towards the future-work possibilities related to my project following significant tasks that I have not covered yet may be:

- Design and implementation of an interfacing device that could translate, store, filter and forward different types of packets to/from any different types of wireless or wired networks. A packet coming from any type of serial bus like CAN must be reformed to travel successfully in any wireless network media and similarly, a packet coming from Wireless media must be again reformed back for its compatibility according to CAN like any type of serial bus.

- Another future step in my project work may be to show a comparative analysis of packet movement in CAN and Wireless LAN by showing graphical chart based outputs. These graphical reports will also cover the load distribution capability within CAN as per CAN's distributed behavior and without CAN under normal wireless packet transferring using different protocols as DSR, AODV, TORA and many more.

- Instead of using IEEE802.11b Wireless LAN, the interfacing bridge-like device may include the latest developed IEEE $802.11 \mathrm{e} / \mathrm{g} / \mathrm{h}$, etc. so that quality of service parameters should also be included. In this way, the performance of the inter-working device will be highly optimized.

- Since nowadays CAN is proving its involvement in aerospace, robotics Homeappliances so In future Controller Area Network protocol should be implemented exhaustively in NS2 like open-source network simulator, so that some other hidden but significant aspects of CAN could be modeled as well as simulated successfully for the creation of new chip-based devices.

\section{REFERENCES}

[1] Vahid, Frank, and Tony Givargis. Embedded system design: a unified hardware/software introduction. Vol. 52. New York: Wiley, 2002.

[2] Pinho, L.M. Vasques, F.M.A. Olson. "Reliable real-time communication in CAN network". IEEE Transactions on Computers, Vol. 52, No. 12, December 2003, pp. 1594-1607.

[3] “CAN specification 2.0”, Parts A and B, Robert Bosch, September 1991.

[4] Farsi, Mohammad, Karl Ratcliff, and Manuel Barbosa. "An overview of controller area network." Computing \& Control Engineering Journal 10.3 (1999): 113-120.

[5] Varshney, Upkar. "The status and future of 802.11-based WLANs." Computer 6 (2003): 102-105.

[6] IEEE Computer Society LAN MAN Standards Committee. "Part 11: Wireless LAN medium access control (MAC) and physical layer (PHY) specifications.

[7] High-speed physical layer in the 5GHz band." IEEE std 802.11 a-1999 (1999).

[8] Cavalieri, S., and D. Panno. "A novel solution to interconnect FieldBus systems using IEEE wireless LAN technology."Computer Standards \& Interfaces 20.1 (1998): 9-23.

[9] I. Eruturk, " Remote access of CAN nodes Used in a Traffic Control Systemto a central Management Unit over Wireless ATM", IEEE $4^{\text {th }}$ International Workshop on Mobile and Wireless Communication Networks, Stockholm, Sweden, 2002, pp. 626-630. 
[10] Wolf, Marilyn. Computers as components: principles of embedded computing system design. Elsevier, 2012.

[11] Eshghi, Farshad, and Ahmed K. Elhakeem. "Performance analysis of ad hoc wireless LANs for realtime traffic." IEEE Journal on Selected areas in communications 21.2 (2003): 204-215.

[12] http://www.Compnetworking.about.com.

[13] Othman, H.F. Aji, Y. R. Fakhreddin, F. T. AL-Ali, A. R. "Controller Area Networks: Evolution and Applications” IEEE Information and Communication technologies, Vol. 2., April 2006, pp. 3088-3093.

[14] http://www.isi.edu/nsnam/ns/.

[15] http://www.isi.edu/nsnam/ns/tutorial.html.

[16] http://www.isi.edu/nsnam/iTCL/doc/tuitorial.html.

[17] P. Nicopolitidis, M. S. Obaidat, G. I. Papadimitriou, A. S. Pomportsis, Wireless Networks. Asia: John Wiley \& Sons, Pvt. Ltd, 2003.

[18] Modieginyane, K. M., Letswamotse, B. B., Malekian, R., \& Abu-Mahfouz, A. M. (2018). Software defined wireless sensor networks application opportunities for efficient network management: A survey. Computers \& Electrical Engineering, 66, 274-287.

[19] Ullo, S., Gallo, M., Palmieri, G., Amenta, P., Russo, M., Romano, G., ... \& De Angelis, M. (2018, March). Application of wireless sensor networks to environmental monitoring for sustainable mobility. In 2018 IEEE International Conference on Environmental Engineering (EE) (pp. 1-7). IEEE.

[20] Muth, M. (2016). U.S. Patent No. 9,471,528. Washington, DC: U.S. Patent and Trademark Office.

[21] Zhang, Y., Chen, M., Guizani, N., Wu, D., \& Leung, V. C. (2017). SOVCAN: Safety-oriented vehicular controller area network. IEEE Communications Magazine, 55(8), 94-99.

[22] I. Eruturk, " Remote access of CAN nodes Used in a Traffic Control System to a central Management Unit over Wireless ATM", IEEE $4^{\text {th }}$ International Workshop on Mobile and Wireless Communication Networks, Stockholm, Sweden, 2002, pp. 626-630. 
International Journal of Security and Its Applications Vol. 13, No. 4 (2019) 